\title{
Summation of divergent series and Borel summability for strongly dissipative differential equations with periodic or quasiperiodic forcing terms
}

\author{
Guido Gentile ${ }^{\text {a) }}$ \\ Dipartimento di Matematica, Universitá di Roma Tre, Roma, I-00146, Italy \\ Michele V. Bartuccelli ${ }^{\text {b) }}$ and Jonathan H. B. Deane ${ }^{\text {c) }}$ \\ Department of Mathematics and Statistics, University of Surrey, Guildford, GU2 7XH, \\ United Kingdom
}

(Received 27 January 2005; accepted 13 April 2005; published online 1 June 2005)

\begin{abstract}
We consider a class of second order ordinary differential equations describing one-dimensional systems with a quasiperiodic analytic forcing term and in the presence of damping. As a physical application one can think of a resistorinductor-varactor circuit with a periodic (or quasiperiodic) forcing function, even if the range of applicability of the theory is much wider. In the limit of large damping we look for quasiperiodic solutions which have the same frequency vector of the forcing term, and we study their analyticity properties in the inverse of the damping coefficient. We find that even the case of periodic forcing terms is nontrivial, as the solution is not analytic in a neighborhood of the origin: it turns out to be Borel summable. In the case of quasiperiodic forcing terms we need renormalization group techniques in order to control the small divisors arising in the perturbation series. We show the existence of a summation criterion of the series in this case also; however, this cannot be interpreted as Borel summability. (C) 2005 American Institute of Physics. [DOI: 10.1063/1.1926208]
\end{abstract}

\section{INTRODUCTION}

Consider the ordinary differential equation

$$
\varepsilon \ddot{x}+\dot{x}+\varepsilon x^{2}=\varepsilon f(\boldsymbol{\omega} t),
$$

where $\boldsymbol{\omega} \in \mathbb{R}^{d}$ is the frequency vector, $f(\boldsymbol{\psi})$ is an analytic function,

$$
f(\boldsymbol{\psi})=\sum_{\boldsymbol{\nu} \in \mathbb{Z}^{d}} e^{i \boldsymbol{\nu} \cdot \psi_{\boldsymbol{\nu}}}
$$

with average $\alpha=a^{2}$, with $a>0$ (hence $\langle f\rangle \equiv f_{0}=\alpha$ ), and $\varepsilon>0$ is a real parameter. Here and henceforth we denote with - the scalar product in $\mathbb{R}^{d}$. By the analyticity assumption of $f$ there are two strictly positive constants $F$ and $\xi$ such that one has $\left|f_{\boldsymbol{\nu}}\right| \leqslant F e^{-\xi|\boldsymbol{\nu}|}$ for all $\boldsymbol{\nu} \in \mathbb{Z}^{d}$.

By writing $\gamma=1 / \varepsilon$ the equation becomes

$$
\ddot{x}+\gamma \dot{x}+x^{2}=f(\boldsymbol{\omega} t),
$$

which describes a nonlinear electronic circuit, known as resistor-inductor-varactor circuit, subject to a quasiperiodic forcing function. Taking $d=1$ and $f(\omega t)=\alpha+\beta \sin t$, this equation has been studied in Ref. 1, where, among other things, it has been found numerically that for $\gamma$ large enough

\footnotetext{
${ }^{\text {a)} E l e c t r o n i c ~ m a i l: ~ g e n t i l e @ m a t . u n i r o m a 3 . i t ~}$

${ }^{b)}$ Electronic mail: m.bartuccelli@surrey.ac.uk

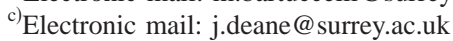


there exists only one attracting periodic orbit and the corresponding period is $2 \pi / \omega=2 \pi$, the same as the forcing term. Furthermore one can prove analytically that such a periodic orbit is the only one in a neighborhood of radius $O(1 / \gamma)$ around the point $(a, 0)$.

Here we give some further analytical support to such numerical findings. In particular we show that, if we take as forcing term an analytic periodic function,

$$
f(\psi)=\sum_{\nu \in \mathbb{Z}} e^{i \nu \psi} f_{\nu}, \quad f_{0}=\alpha>0,
$$

then for $\varepsilon$ small enough there is a $2 \pi / \omega$-periodic solution, but this is not analytic in $\varepsilon=1 / \gamma$ in a neighborhood the origin in the complex $\varepsilon$-plane. We find that such a solution is Borel summable.

We also show that by considering quasiperiodic forcing terms, as in (1.3), we still have a quasiperiodic solution with the same frequency vector $\boldsymbol{\omega}$ as the forcing term, but we can only say in general that such a solution is analytic in a domain with boundary crossing the origin.

Finally we shall see that considering more general nonlinearities introduces no further difficulties, and equations like

$$
\ddot{x}+\gamma \dot{x}+g(x)=f(\boldsymbol{\omega} t), \quad \lim _{|x| \rightarrow \infty} \frac{|g(x)|}{|x|}=\infty,
$$

with $g$ and $f$ both analytic in their arguments, can be dealt with essentially in the same way. Simply, we must impose a nondegeneracy condition on the function $g$, which reads as

$$
\exists x_{0} \text { such that } g\left(x_{0}\right)=f_{\mathbf{0}} \text { and } g^{\prime}\left(x_{0}\right) \neq 0 .
$$

In the particular case of homogeneous $g(x)$, that is $g(x)=\sigma x^{p}$, with $p \geqslant 2$ an integer and $\sigma \in \mathbb{R}$, the condition is automatically satisfied if $p$ is odd (for any value of $\sigma$ ), while it requires $\sigma f_{0}>0$ for $p$ even, as assumed in (1.1).

The paper is organized as follows. For expository clearness we start with the case of periodic forcing terms. In Secs. II and III we show that a periodic solution with frequency $\omega$ in the form of a formal power series in $\varepsilon$ (perturbation series) is well defined to all orders, and it admits a natural graphical representation. In Sec. IV we study further such a series, and we see that there is strong evidence to show that it diverges (even if we cannot exclude convergence definitely). The best bounds that we are able to provide for the coefficients grow as factorials. To obtain bounds which allow summability of the perturbation series we must perform a suitable summation in order to give the series a meaning. This is done in Sec. V, and the resummed series is found to represent a $2 \pi / \omega$-periodic solution which is Borel summable in $\varepsilon$. To prove the latter property we rely on Nevanlinna's improvement of Watson's theorem. ${ }^{12}$ In Sec. VI we consider the case of quasiperiodic forcing terms. We find that the perturbation series is well defined if the frequency vector of the forcing term satisfies a Diophantine condition, and, by using renormalization group techniques in order to deal with the small divisors problem, we find that the resummed series still converges to a quasiperiodic solution, and it defines a function analytic in a domain containing the origin in its boundary. We shall see that the bounds we find do not allow us any more to obtain Borel summability, unlike the case of periodic forcing terms. In Sec. VII we discuss how to extend the analysis to more general nonlinearities $g(x)$, by requiring the condition (1.6) to be satisfied.

The interest of the approach we propose is that it allows the use of perturbation theory which can be very natural in problems in which a small parameter appears. In fact analyticity in $\varepsilon$ for $\varepsilon$ close to 0 (that is in $\gamma$ for $\gamma$ large enough) could be proved very likely with other techniques (at least for periodic solutions in the case of periodic forcing terms), but a naive expansion in powers of $\varepsilon$ is prevented by the lack of analyticity in a neighborhood of the origin. On the other hand, the perturbation series gives a very accurate description of the solution, hence it is important to know that such a series is an asymptotic series, and its use is fully justified. Finally we mention that the quasiperiodic solution we investigate is of physical relevance, hence it is useful to study its 
properties. For instance in the case of the aforementioned resistor-inductor-varactor circuit in Ref. 1 , for damping large enough, the $2 \pi / \omega$-periodic solution is numerically found to attract any trajectory which remains bounded in phase space.

The techniques we use have been recently developed for problems of Hamiltonian stability, and are based on resummation methods that are familiar in quantum field theory (see Ref. 7 and references quoted therein). Here we show that they can be useful even in non-Hamiltonian problems with viscosity acting. We leave as an open problem to show whether the formal series of the periodic or quasiperiodic solutions are really divergent. We also note that we are not able to prove uniqueness of the quasiperiodic solutions we find by the resummation procedure, as in that case there is no uniqueness result as for analytic or Borel summable functions which one can rely upon. Furthermore, both for periodic and for quasiperiodic solutions, we cannot exclude existence of other solutions with the same rotation vector, which either are of a different form or even admit the same formal series, without being obtained through the same resummation procedure. Problems of the same kind were met in the study of hyperbolic lower-dimensional tori. ${ }^{6}$

\section{FORMAL ANALYSIS}

Consider first (1.1) for $d=1$, that is

$$
\varepsilon \ddot{x}+\dot{x}+\varepsilon x^{2}=\varepsilon f(\omega t),
$$

with $f(\psi)$ given by (1.4). We look for bounded solutions (if any) which are analytic in $\varepsilon$, that is of the form

$$
x(t)=\sum_{k=0}^{\infty} \varepsilon^{k} x^{(k)}(t) .
$$

Inserting (2.2) into (2.1) and equating terms of the same Taylor order we find the set of recursive equations

$$
\begin{gathered}
\dot{x}^{(0)}=0, \\
\dot{x}^{(1)}=-\ddot{x}^{(0)}-x^{(0) 2}+f, \\
\dot{x}^{(k)}=-\ddot{x}^{(k-1)}-\sum_{k_{1}+k_{2}=k-1} x^{\left(k_{1}\right)} x^{\left(k_{2}\right)}, \quad k \geqslant 2 .
\end{gathered}
$$

From the first equation (zeroth order) we obtain that $x^{(0)}$ must be constant, say $x^{(0)}=c_{0}$ with $c_{0}$ to be determined. The second equation (first order) can give a bounded solution only if $-c_{0}^{2}+\alpha=0$, which fixes $c_{0}=\sqrt{\alpha}=a$ and gives $x^{(1)}(t)$ as a periodic function with the same period of the forcing term,

$$
x^{(1)}(t)=x^{(1)}(0)+\int_{0}^{t} \mathrm{~d} t^{\prime}\left(f\left(\omega t^{\prime}\right)-\alpha\right) .
$$

As each $x^{(k)}(t)$ depends on the functions $x^{\left(k^{\prime}\right)}(t)$ with $k^{\prime}<k$, we expect that if there is any periodic solution then it must have the same period as the forcing term.

To continue the analysis to all orders it is more convenient to write the recursive equations (2.3) in Fourier space. The analysis to first order and the considerations above motivate us to write in $(2.2)$,

$$
x(t)=\sum_{k=0}^{\infty} \varepsilon^{k} x^{(k)}(t)=\sum_{k=0}^{\infty} \varepsilon^{k} \sum_{\nu \in \mathbb{Z}} e^{i \nu \omega t} x_{\nu}^{(k)},
$$

which inserted into (2.3) gives for $\nu \neq 0$, 


$$
\begin{gathered}
x_{\nu}^{(0)}=0, \\
x_{\nu}^{(1)}=\frac{f_{\nu}}{i \omega \nu}, \\
x_{\nu}^{(k)}=-(i \omega \nu) x_{\nu}^{(k-1)}-\frac{1}{i \omega \nu} \sum_{\substack{k_{1}+k_{2}=k-1 \\
k_{1}, k_{2} \geqslant 0}} \sum_{\substack{\nu_{1}+\nu_{2}=\nu \\
\nu_{1}}} x_{\nu_{1}}^{\left(k_{1}\right)} x_{\nu_{2}}^{\left(k_{2}\right)}, \quad k \geqslant 2,
\end{gathered}
$$

provided that one has for $\nu=0$,

$$
\begin{gathered}
0=-x_{0}^{(0) 2}+f_{0}, \\
0=\sum_{\substack{k_{1}+k_{2}=k \\
k_{1}, k_{2} \geqslant 0}} \sum_{\substack{\nu_{1}+\nu_{2}=0 \\
\nu_{1}}} x_{\left.\nu_{1}\right)}^{\left(k_{1}\right)} x_{\nu_{2}}^{\left(k_{2}\right)}, k \geqslant 1 .
\end{gathered}
$$

If we set $x_{0}^{(k)}=c_{k}$ then the first of (2.7) fixes, as already noted,

$$
c_{0}=a=\sqrt{\alpha},
$$

because one has $f_{0}=\alpha>0$, while the second of (2.7) gives

$$
\sum_{k^{\prime}=0}^{k} \sum_{\nu_{1} \in Z} x_{\nu_{1}}^{\left(k-k^{\prime}\right)} x_{-\nu_{1}}^{\left(k^{\prime}\right)}=0 .
$$

The latter equation, by taking into account (2.8) and the first of (2.6), can be more conveniently written as

$$
c_{1}=0, \quad c_{k}=-\frac{1}{2 c_{0}} \sum_{k^{\prime}=1}^{k-1} \sum_{\nu_{1} \in \mathbb{Z}} x_{\nu_{1}}^{\left(k-k^{\prime}\right)} x_{-\nu_{1}}^{\left(k^{\prime}\right)}, \quad k \geqslant 2,
$$

which provides an iterative definition of the coefficients $c_{k}$ as the right-hand side depends only on the coefficients $c_{k^{\prime}}$ with $k^{\prime}<k$. To deduce $c_{1}=0$ we used the first of (2.6), which, inserted into (2.9) for $k=1$, gives $2 c_{0} c_{1}=0$, hence $c_{1}=0$ as $c_{0} \neq 0$.

The following result holds.

Lemma 2.1: Consider (2.1) with $f$ given by (1.4). Then there exists a formal power series solution (2.2) whose coefficients $x^{(k)}(t)$ are analytic in $t$. If $f$ is a trigonometric polynomial, that is in (1.4) one has $|\nu| \leqslant N$ for some $N \in \mathbb{N}$, then for all $k \geqslant 0$ the functions $x^{(k)}(t)$ are trigonometric polynomials of order $[(k+1) / 2] N$, where $[\cdot]$ denotes the integer part. This means that one has $x_{\nu}^{(2 k)}=0$ and $x_{\nu}^{(2 k-1)}=0$ for $|\nu|>k N$.

Proof: The existence of a formal solution (2.2), with coefficients $x^{(k)}(t)$ analytic in $t$ for all $k \geqslant 0$, follows from the analysis above. If $f$ is a trigonometric polynomial of degree $N$, that the coefficients $x_{v}^{(k)}$ are trigonometric polynomials with the stated properties can be proved from (2.6) by induction on $k$.

Then the functions $x^{(k)}(t)$ are well defined to all orders. Before discussing the issue of convergence of the formal power series defining such functions we look for a graphical representation of the coefficients $x_{v}^{(k)}$.

\section{GRAPHICAL REPRESENTATION AND TREE FORMALISM}

We start by giving some abstract definitions.

Definition 3.1 (trees): A tree $\theta$ is a graph, that is a connected set of points and lines, with no 
cycle, such that all the lines are oriented toward a unique point which has only one incident line. Such a point is called the root of the tree. All the points in a tree except the root are denoted nodes. The line entering the root is called the root line. The orientation of the lines in a tree induces a partial ordering relation between the nodes. We denote this relation by $\leqslant$, given two nodes $v$ and $w$, we shall write $w \leqslant v$ every time $v$ is along the path (of lines) which connects $w$ to the root.

Given a tree $\theta$, we can identify the following subsets in $\theta$.

Definition 3.2 (endpoints): We call $E(\theta)$ the set of endpoints in $\theta$, that is the nodes which have no entering line. The endpoints can be represented either as white bullets or as black bullets. We call $E_{W}(\theta)$ the set of white bullets and $E_{B}(\theta)$ the set of black bullets. Of course $E_{W}(\theta) \cup E_{B}(\theta)$ $=E(\theta)$. With each $v \in E_{W}(\theta)$ we associate a mode label $\nu_{v}=0$, an order label $k_{v} \in \mathbb{Z}_{+}$and a node factor $F_{v}=c_{k_{v}}$. With each $v \in E_{B}(\theta)$ we associate a mode label $\nu_{v} \in \mathbb{Z} \backslash\{0\}$, and a node factor $F_{v}$ $=f_{\nu_{v}}$.

Definition 3.3 (lines): We denote with $L(\theta)$ the set of lines in $\theta$. Each line $\ell \in L(\theta)$ leaves a point $v$ and enters another one which we shall denote by $v^{\prime}$. Since $\ell$ is uniquely identified with $v$ (the point which $\ell$ leaves), we may write $\ell=\ell_{v}$. With each line $\ell$ we associate a momentum label $\nu_{\ell} \in \mathbb{Z}$ and a propagator

$$
g_{\ell}= \begin{cases}1 /\left(i \omega \nu_{\ell}\right), & \nu_{\ell} \neq 0, \\ 1, & \nu_{\ell}=0,\end{cases}
$$

and we say that the momentum $\nu_{\ell}$ flows through the line $\ell$. The modes and the momenta are related as follows: if $\ell=\ell_{v}$ one has

$$
\nu_{\ell}=\sum_{i=1}^{s_{v}} \nu_{\ell_{i}}=\sum_{\substack{w \in E_{B}(\theta) \\ w \leqslant v}} \nu_{w}
$$

where $\ell_{1}, \ldots, \ell_{s_{v}}$ are the lines entering $v$.

Definition 3.4 (vertices): We denote by $V(\theta)$ the set of vertices in $\theta$, that is the set of points which have at least one entering line. If $V(\theta) \neq \varnothing$ we call the vertex $v_{0}$ connected to the root the last vertex of the tree. If $s_{v}$ denotes the number of lines entering $v$ call $\max _{v \in V(\theta)} s_{v}$ the branching number. One can have either $s_{v}=1$ or $s_{v}=2$. We set $V_{s}(\theta)=\left\{v \in V(\theta): s_{v}=s\right\}$ for $s=1,2$; of course $V_{1}(\theta) \cup V_{2}(\theta)=V(\theta)$. We define also $V_{0}(\theta)=\left\{v \in V(\theta): \nu_{\ell_{v}}=0\right\}$; one has $V_{0}(\theta) \subset V_{2}(\theta)$. We require that either $V_{0}(\theta)=\varnothing$ or $V_{0}(\theta)=\left\{v_{0}\right\}$, and that one can have $v \in V_{1}(\theta)$ only if $\nu_{\ell_{v}} \neq 0$. We associate with each vertex $v \in V(\theta)$ a node factor

$$
F_{v}=\left\{\begin{array}{lll}
-1, & s_{v}=2 \\
-1 / 2 c_{0}, & s_{v}=2 \\
-\left(i \omega \nu_{\ell_{v}}\right)^{2}, & s_{v}=1,
\end{array} \text { and } v \notin V_{0}(\theta),\right.
$$

which is always well defined as $c_{0} \neq 0$.

We call equivalent two trees which can be transformed into each other by continuously deforming the lines in such a way that they do not cross each other.

Let $\mathcal{T}_{k, \nu}$ be the set of inequivalent trees $\theta$ such that

(1) the number of vertices, the number of black bullets, and the order labels of the white bullets are such that we have

$$
\begin{gathered}
k_{1}+k_{2}+k_{3}=k, \quad \text { if } \nu \neq 0, \\
k_{1}+k_{2}+k_{3}=k+1, \quad \text { if } \nu=0,
\end{gathered}
$$

if we set $k_{1}=|V(\theta)|, k_{2}=\left|E_{B}(\theta)\right|$, and $k_{3}=\sum_{v \in E_{W}(\theta)} k_{v}$.

(2) The momentum flowing through the root line is $\nu$. 


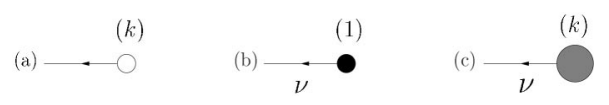

FIG. 1. Graphical representation of $x_{0}^{(k)}, x_{\nu}^{(1)}$, and $x_{\nu}^{(k)}$. For $\nu=0$ the latter reduces to the first graph, while for $k=1$ and $\nu$ $\neq 0$ it reduces to the second graph. In the first graph the momentum is not shown as it is necessarily $\nu=0$.

We refer to $\mathcal{T}_{k, \nu}$ as the set of trees of order $k$ and total momentum $\nu$.

With the above definitions the following result holds.

Lemma 3.5: For all $k \geqslant 1$ and all $\nu \neq 0$ one has

$$
x_{\nu}^{(k)}=\sum_{\theta \in \mathcal{T}_{k, \nu}} \operatorname{Val}(\theta), \quad \operatorname{Val}(\theta)=\left(\prod_{\ell \in L(\theta)} g_{\ell}\right)\left(\prod_{v \in E(\theta) \cup V(\theta)} F_{v}\right),
$$

where $\mathrm{Val}: \mathcal{T}_{k, \nu} \rightarrow \mathrm{C}$ is called the value of the tree. For $k \geqslant 2$ and $\nu=0$ one has

$$
x_{0}^{(k)} \equiv c_{k}=\sum_{\theta \in \mathcal{T}_{k, 0}}{ }^{*} \operatorname{Val}(\theta),
$$

where $*$ means that there are two lines entering the last vertex $v_{0}$ of $\theta_{0}$ and neither one exits from an endpoint $v$ with order label $k_{v}=0$.

Proof: We can represent graphically $x_{0}^{(k)}=c_{k}$ as in Fig. 1(a), $x_{\nu}^{(1)}, \nu \neq 0$, as in Fig. 1(b), and, more generally, $x_{v}^{(k)}$ as in Fig. 1(c).

Then the third equation in (2.6) can be represented graphically as in Fig. 2, if we associate with the nodes and to the lines the node factors and the propagators, respectively, according to the definitions (3.1) and (3.3).

Analogously (2.10) is represented graphically as in Fig. 3, again if we use the graphical representations in Fig. 1 and associate with the lines and vertices the propagators (3.1) and the node factors (3.3), respectively.

Note that in this way we represent graphically each coefficient $x_{\nu}^{(k)}$ in terms of other coefficients $x_{v^{\prime}}^{\left(k^{\prime}\right)}$, with $k^{\prime}<k$, so that we can apply iteratively the graphical representation in Fig. 2 until only trees whose endpoints represent either $x_{\nu}^{(1)}$ with $\nu \neq 0$ (black bullets) or $c_{k}$ are left (white bullets). This corresponds exactly to the expressions in (3.5) and (3.6).

To get familiar with the graphical representation (3.5) and (3.6) one should try to draw the trees which correspond to the first orders, and check that the sum of the values obtained with the graphical rules listed above gives exactly the same analytical expression which can be deduced directly from (2.6) and (2.10).

For instance for $k=2$ we obtain for $x_{\nu}^{(2)}, \nu \neq 0$, the graphical representation in Fig. 4 and for $c_{2}=x_{0}^{(2)}$ the graphical representation in Fig. 5 .

For $k=3$ we obtain for $x_{\nu}^{(3)}, \nu \neq 0$, the graphical representation in Fig. 6 and for $c_{3}=x_{0}^{(3)}$ the graphical representation in Fig. 7, where we have explicitly used that $c_{1}=0$.

This can be continued to higher orders. In general a tree $\theta \in \mathcal{T}_{k, \nu}$ looks like in Fig. 8, where for simplicity no labels have been drawn other than the order labels of the white bullets. Note that each node can have only one or two entering lines, while the endpoints have no entering line at all. Moreover the momentum flowing through the line exiting a vertex $v$ is equal to the sum of the

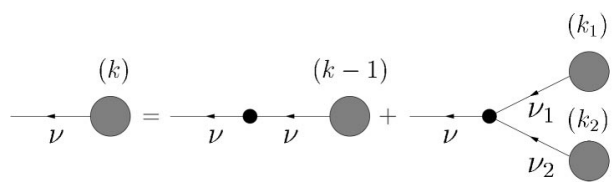

FIG. 2. Graphical representation of the third equation in (2.6) expressing the coefficient $x_{\nu}^{(k)}$ for $k \geqslant 2$ and $\nu \neq 0$ in terms of the coefficients $x_{\nu^{\prime}}^{\left(k^{\prime}\right)}$ with $k^{\prime}<k$. In the last graph one has the constraints $k_{1}+k_{2}=k-1$ and $\nu_{1}+\nu_{2}=\nu$. 


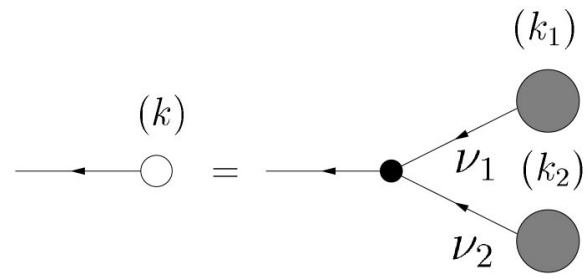

FIG. 3. Graphical representation of the equation (2.10) expressing the coefficient $c_{k}$ for $k \geqslant 2$ in terms of the coefficients $x_{\nu^{\prime}}^{\left(k^{\prime}\right)}$ with $k^{\prime}<k$. Both $k_{1}$ and $k_{2}$ are strictly positive and $k_{1}+k_{2}=k$; moreover $\nu_{1}+\nu_{2}=0$.

momenta flowing through the lines entering $v$, according to (3.2); this is a sort of conservation law. The order of the tree is given by the number of vertices and black bullets plus the sum of the order labels of the white bullets minus the number of vertices in $V_{0}(\theta)$. The latter is just $\left|V_{0}(\theta)\right|=0$ if $\theta \in \mathcal{T}_{k, \nu}, \nu \neq 0$, and $\left|V_{0}(\theta)\right|=1$ if $\theta \in \mathcal{T}_{k, 0}$.

If a vertex $v$ has $s_{v}=1$, that is it has only one entering line $\ell$, the latter cannot come out of a white bullet. Indeed if this occurs one should have $\nu_{\ell_{v}}=\nu_{\ell}=0$, hence $F_{v}=0$ by (3.3), so that the value of the tree containing such a vertex is zero.

Given a tree as in Fig. 8 we can represent each white bullet according to the graphical representation in Fig. 3, corresponding to the analytic formula (3.6), and expand again the two contributions $x_{\nu_{1}}^{\left(k_{1}\right)}$ and $x_{\nu_{2}}^{\left(k_{2}\right)}$ as sums of trees, and so on, iteratively, until the only white bullets which are left are the ones with order label $k=0$. In this way we obtain a new graphical representation where the trees still look like those in Fig. 8, but now there are a few differences as follows:

(1) all the white bullets $v \in E_{W}(\theta)$ have order labels $k_{v}=0$, and

(2) there can be lines $\ell \in L(\theta)$ with momentum $\nu_{\ell}=0$ which come out of vertices, that is $V_{0}(\theta)$ can contain no element or more than one element.

Note that only lines coming out either from nodes in $V_{0}(\theta) \subset V_{2}(\theta)$ or from white bullets have vanishing momentum.

The order of the tree is then given by the number of elements of $V(\theta) \cup E_{B}(\theta)$ minus the number of elements of $V_{0}(\theta)$, that is $k=|V(\theta)|+\left|E_{B}(\theta)\right|-\left|V_{0}(\theta)\right|$. Of course $v_{0} \in V_{0}(\theta)$ if and only if the momentum of the root line is vanishing, that is $\theta \in \mathcal{T}_{k, 0}$ for some $k \geqslant 2$. It is important to stress that no line entering a vertex $v \in V_{0}(\theta)$ can come out of a white bullet (which now has necessarily an order label 0), because this would be against the constraint in the sum (3.6). This means that if two lines carrying zero momentum enter the same vertex $v$ [so that $v \in V_{0}(\theta)$ according to (3.2)], then none of them can exit from a white bullet.

But up to these minor differences a tree representation like in (3.5) and (3.6) still holds. The advantage of these modified rules is that now the tree values are expressed no longer in terms of constants $c_{k}$ to be determined, but only in terms of $c_{0}$ which is known. A tree drawn according these new rules is represented as in Fig. 8 with $k_{1}=k_{2}=k_{3}=0$ (and in particular a tree of this kind can contribute only to $x_{\nu}^{(k)}$ with $\nu \neq 0$ ). Note that we could avoid drawing the order labels associated with the endpoints, as they are uniquely determined as $k=0$ for the white bullets and $k=1$ for the black bullets. Of course, with respect to the caption of that figure, now the order $k$ is given by the number of elements in $V(\theta)$ plus the number of elements in $E_{B}(\theta)$ minus the number of elements in $V_{0}(\theta)$.

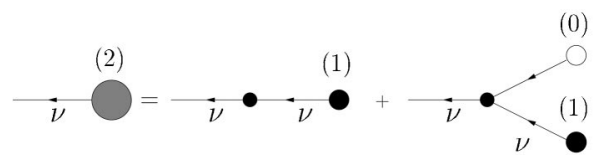

FIG. 4. Graphical representation of $x_{v}^{(2)}$ for $\nu \neq 0$. The second contribution must be counted twice, because there is also a tree with the white and black bullets exchanged; of course the latter has the same value. 


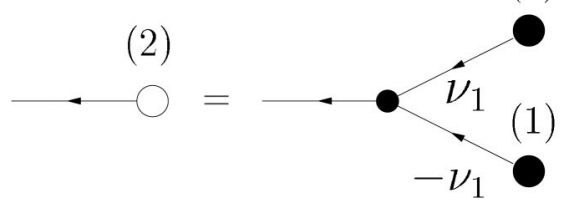

FIG. 5. Graphical representation of $c_{2}=x_{0}^{(2)}$. There is no contribution with any white bullet carrying order label $k=0$ and $k=1$ because of the restriction in the sum appearing in (3.6) and of the fact that $c_{1}=0$, respectively.

\section{FORMAL SOLUTIONS}

The sum over the trees in (3.5) and (3.6), with the new definition of the set $\mathcal{T}_{k, \nu}$ given at the end of Sec. II, can be performed by summing over all possible "tree shapes" (that is trees without labels or unlabeled trees) and, for a fixed shape, over all possible assignments of mode labels. In the case of a trigonometric polynomial of degree $N$ the latter can be bounded by $(2 N)^{|E(\theta)|}$, because each endpoint $v$ can have either a mode label $\nu_{v} \neq 0$, with $\left|\nu_{v}\right| \leqslant N$, or the mode label $\nu_{v}=0$, while the case of analytic functions (or even to obtain bounds which are uniform in $N$ ) must be discussed a little more carefully. The number of unlabeled trees with $P$ nodes (vertices and endpoints) can be bounded by $2^{2 P}$.

Recall that $V_{s}(\theta)$ denotes the set of vertices $v$ such that $s_{v}=s$; of course $V_{1}(\theta) \cup V_{2}(\theta)=V(\theta)$, and $V_{0}(\theta) \subset V_{2}(\theta)$. Analogously we can set

$$
\begin{gathered}
L_{0}(\theta)=\left\{\ell \in L(\theta): n_{\ell}=0,\right\}, \\
L_{1}(\theta)=\left\{\ell \in L(\theta): \ell=\ell_{v}, v \in V_{1}(\theta)\right\}, \\
L_{2}(\theta)=L(\theta) \backslash\left(L_{0}(\theta) \cup L_{1}(\theta)\right),
\end{gathered}
$$

with the splitting made in such a way that one has

$$
\begin{aligned}
& \left|\prod_{v \in V_{1}(\theta)} F_{v}\right|\left|\prod_{\ell \in L_{1}(\theta)} g_{\ell}\right| \leqslant \prod_{\ell \in L_{1}(\theta)}\left|\omega \nu_{\ell}\right|, \quad\left|\prod_{\ell \in L_{2}(\theta)} g_{\ell}\right| \leqslant \prod_{\ell \in L_{2}(\theta)} \frac{1}{\left|\omega \nu_{\ell}\right|}, \\
& \left|\prod_{v \in V_{0}(\theta)} F_{v}\right| \leqslant\left(\frac{1}{2 c_{0}}\right)^{\left|V_{0}(\theta)\right|},\left|\prod_{v \in E_{W}(\theta)} F_{v}\right| \leqslant c_{0}^{\left|E_{W}(\theta)\right|},
\end{aligned}
$$

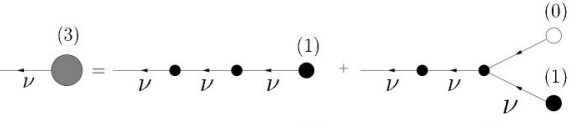

$$
\begin{aligned}
& \nu_{\nu}^{\bullet} \bullet_{\nu}^{(1)} \bullet_{(1)}^{(0)} \bigodot_{\nu}^{(0)} \bullet_{\nu}^{(1)}
\end{aligned}
$$

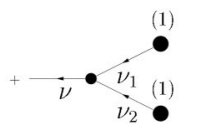

FIG. 6. Graphical representation of $x_{\nu}^{(3)}$ for $\nu \neq 0$. The second and fourth contributions must be counted twice, while the third one must be counted four times. There is no contribution with any white bullet carrying the order label $k=1$ as $c_{1}$ $=0$. 


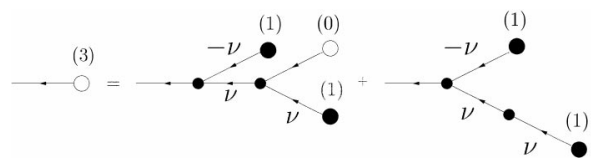

FIG. 7. Graphical representation of $c_{3}=x_{0}^{(3)}$. The second contribution must be counted twice, while the first one must be counted four times. There is no contribution with any white bullet carrying the order label $k=1$ as $c_{1}=0$.

$$
\left|\prod_{v \in E_{B}(\theta)} F_{v}\right| \leqslant F^{\left|E_{B}(\theta)\right|} \prod_{v \in E_{B}(\theta)} e^{-\xi\left|\nu_{v}\right|}
$$

where for each line $\ell$ one has $\left|\nu_{\ell}\right| \leqslant \Sigma_{v \in E_{W}(\theta)}\left|\nu_{v}\right|$.

The following result is useful when looking for bounds on the tree values.

Lemma 4.1: Given a tree $\theta$ with branching number $s$ one has $|E(\theta)| \leqslant(s-1)|V(\theta)|+1$. If $k$ denotes the order of the tree $\theta$, that is $|V(\theta)|-\left|V_{0}(\theta)\right|+\left|E_{B}(\theta)\right|=k$, one has the identity $\left|L_{1}(\theta)\right|$ $+\left|L_{2}(\theta)\right|=k$, and the bounds $\left|V_{1}(\theta)\right| \leqslant k,\left|V_{0}(\theta)\right| \leqslant k-1,|E(\theta)| \leqslant k$ and $|E(\theta)|+|V(\theta)| \leqslant 2 k-1$.

Proof: It is a standard result on trees that one has $\Sigma_{v \in V(\theta)}\left(s_{v}-1\right)=|E(\theta)|-1$, so that the first bound follows. The bounds on $\left|V_{1}(\theta)\right|,\left|V_{0}(\theta)\right|,|E(\theta)|$ and $|E(\theta)|+|V(\theta)|$ can be easily proved by induction, while the identity $\left|L_{1}(\theta)\right|+\left|L_{2}(\theta)\right|=k$ follows from the observation that all lines in $L_{1}(\theta)$ and $L_{2}(\theta)$ come out either of vertices or of black bullets, and they have nonvanishing momentum.

Hence the number of lines in $L_{1}(\theta)$ is bounded by $k$, so that in (4.2) we can bound

$$
\begin{aligned}
\left(\prod_{\ell \in L_{1}(\theta)}\left|\omega \nu_{\ell}\right|\right)\left(\prod_{v \in E_{B}(\theta)} F e^{-\xi\left|\nu_{v}\right|}\right) & \leqslant\left(\prod_{v \in E_{B}(\theta)} F e^{-\xi\left|\nu_{v}\right| 2}\right)\left(\prod_{\ell \in L_{1}(\theta)} e^{\left.-\xi\left|\nu_{\ell}\right| 2 k\left|\omega \nu_{\ell}\right|\right)}\right. \\
& \leqslant\left(\prod_{v \in E_{B}(\theta)} F e^{-\xi\left|\nu_{v}\right| 2}\right)\left(\frac{2 k|\omega|}{\xi}\right)^{k},
\end{aligned}
$$

and in the second line the product can be used to perform the sum over the Fourier labels-this gives a factor $F^{k} B_{2}^{k}$, with $B_{2}=2 e^{-\xi / 2}\left(1-\mathrm{e}^{-\xi / 2}\right)^{-1}$ — while the last factor is bounded by $A_{1} B_{1}^{k} k$ !, for some constants $A_{1}$ and $B_{1}$.

We can bound the value of a tree $\theta$ by using the bounds (4.2) and (4.3), and Lemma 4.1. If we define

$$
\varepsilon_{1}^{-1}=\max \left\{B_{1},|\omega|^{-1}\right\} \max \left\{c_{0}, F B_{2}\right\} \max \left\{1,\left(2 c_{0}\right)^{-1}\right\},
$$

with $c_{0}=\sqrt{\alpha}$, and take into account that the number of unlabeled trees in $\mathcal{T}_{k, \nu}$ is bounded by $2^{2 k-1}$ (because each tree in $\mathcal{T}_{k, \nu}$ has at most $2 k-1$ nodes), then

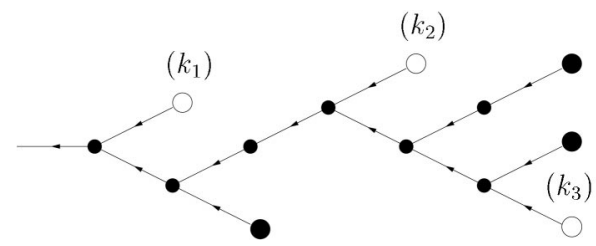

FIG. 8. Example of tree appearing in the graphical expansions (3.5) and (3.6). The number of lines entering any vertex $v$ can be only either $s_{v}=1$ or $s_{v}=2$, while no line enters the endpoints. The order of the tree is given by the number of elements in $V(\theta) \backslash V_{0}(\theta)$ plus the number of elements in $E_{B}(\theta)$ plus the sum of the order labels of the white bullets. Then, if $k_{1}, k_{2}$, and $k_{3}$ are the order labels of the white bullets in the figure, the order of the tree is $k=k_{1}+k_{2}+k_{3}+10$ if $v_{0}$ $\in V_{0}(\theta)$ and $k=k_{1}+k_{2}+k_{3}+9$ if $v_{0} \notin V_{0}(\theta)$. In the latter case one must have $k_{1}>0$ because of the constraint in the sum appearing in (3.6). 


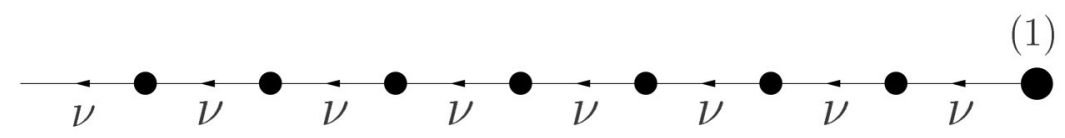

FIG. 9. Example of tree whose value grows as a factorial. If $k$ is the order of the tree (hence there are $k-1$ vertices and 1 black bullet), then the value of the tree is given in (4.6).

$$
\left|x_{\nu}^{(k)}\right| \leqslant A_{1} \varepsilon_{2}^{-k} k !, \quad\left|x^{(k)}(t)\right| \leqslant A_{1} \varepsilon_{2}^{-k} k !,
$$

where we have set $\varepsilon_{2}=\varepsilon_{1} 2^{-2}$.

A bound like (4.5) is obtained also in the case of forcing terms which are trigonometric polynomials, because in general we can bound the factors $\left|\omega \nu_{\ell}\right|$ in (4.2) only with $k N$ (see Lemma 2.1 ), and this produces an overall bound proportional to $k$ ! Note that in that case the bound $B_{2}$, arising from the sum over the Fourier labels, can be replaced with a factor $2 N$, and $B_{1}$ can be replaced with $|\omega| N$.

Then we have proved the following result.

Proposition 4.2: Given the equation (2.1) with $f$ as in (1.4), there is only one periodic solution in the form of a formal power series, and the corresponding period is the same period $2 \pi / \omega$ as the forcing term. The coefficients of such a formal power series satisfy the bounds (4.5).

One could ask if the factorials arising in the bounds are only a technical problem, or whether they are a sign that the series really diverges. To order $k$ one can easily provide examples of trees which grow like factorials; see for instance the tree represented in Fig. 9, where there are $k-1$ vertices with only one entering line. Then the corresponding value is

$$
\operatorname{Val}(\theta)=(i \omega \nu)^{2(k-1)} \frac{1}{(i \omega \nu)^{k}} f_{\nu}=(i \omega \nu)^{k-2} f_{\nu},
$$

which behaves as $k$ ! for large $k$. Furthermore it is unlikely that there are cancellations with the values of other trees because the value of any other tree $\theta \in \mathcal{T}_{k, \nu}$ can be proportional at most to $(i \omega \nu)^{p}$, with $p<k-2$ (strictly). Hence we expect that the coefficients $u_{\nu}^{(k)}$, even if well defined to all orders, grow like factorials, so preventing the convergence of the series.

The lack of analyticity is further supported by the following fact. If we consider (2.1) without the quadratic term and with $\alpha=0$, that is

$$
\varepsilon \ddot{x}+\dot{x}=f(\omega t), \quad f_{0}=0,
$$

in Fourier space, we find $x_{0}=0$ and $i \omega \nu(1+i \varepsilon \omega \nu) x_{\nu}=f_{\nu}$ for $\nu \neq 0$. Hence the equation is trivially solvable, and it gives

$$
x(t)=\sum_{\nu \neq 0} \frac{f_{\nu}}{i \omega \nu(1+i \varepsilon \omega \nu)} e^{i \omega \nu t} .
$$

Of course the solution $x(t)$ of the linear equation is not analytic in $\varepsilon$ (in a neighborhood of the origin) when $f$ is an analytic function containing all the harmonics, as each point $\varepsilon=i / \omega \nu$ represents a singularity point for $x(t)$, and such points accumulate to the origin as $\nu \rightarrow \infty$. Then it is likely that also when the quadratic terms are taken into account the solution cannot be analytic. Therefore giving a meaning to the perturbation series requires some more work, and we discuss this next.

An important remark is that for any $k \geqslant 1$ there is no tree whose value can be bounded worse than proportionally to a factorial, as the estimates (4.5) show, indeed they have been obtained by bounding separately the value of each single tree. This observation will play an important role in the forthcoming analysis. 


\section{PERIODIC FORCING TERMS}

To deal completely with the case of analytic functions and prove existence of the periodic solution, we must modify the graphical expansion envisaged in the preceding sections.

Let us come back to the equation (2.1), and write it in Fourier space. For $\nu \neq 0$ and denoting with $x_{\nu}$ the $\nu$ th Fourier coefficient, we obtain

$$
\varepsilon(i \omega \nu)^{2} x_{\nu}+i \omega \nu x_{\nu}+\varepsilon \sum_{\nu_{1}+\nu_{2}=\nu} x_{\nu_{1}} x_{\nu_{2}}=\varepsilon f_{\nu},
$$

provided that for $\nu=0$ we have

$$
\sum_{\nu_{1}+\nu_{2}=0} x_{\nu_{1}} x_{\nu_{2}}=0
$$

Let us rewrite (5.1) as

$$
\varepsilon(i \omega \nu)^{2} x_{\nu}+i \omega \nu x_{\nu}+\mu \varepsilon \sum_{\nu_{1}+\nu_{2}=\nu} x_{\nu_{1}} x_{\nu_{2}}=\mu \varepsilon f_{\nu},
$$

and look for a solution $x(t)$ which is analytic in $\mu$, which suggests us to write

$$
x(t)=\sum_{k=0}^{\infty} \mu^{k} x^{[k]}(t) .
$$

Of course we want the value $\mu=1$ to be inside the analyticity domain. Note also that now $x^{[k]}$, the coefficient to order $k$, has a different meaning with respect to the previous expansion (1.4) in powers of $\varepsilon$, and for this reason with use a different symbol to denote it. We shall call the series (5.4) the resummed series, because the coefficients $x^{[k]}(t)$ depend on $\varepsilon$, and are given by the sum of infinitely many terms of the formal series (2.5).

Again for $k=0$ we must take $x_{\nu}^{[0]}=0$ for $\nu \neq 0$ and fix $c_{0} \equiv x_{0}^{[0]}=\sqrt{\alpha}$, with $\alpha \equiv f_{0}$.

To order $k \geqslant 1$ (in $\mu$ ) we obtain for $\nu \neq 0$,

$$
i \omega \nu(1+i \varepsilon \omega \nu) x_{\nu}^{[k]}=\varepsilon f_{\nu} \delta_{k, 1}-\varepsilon \sum_{k_{1}+k_{2}=k-1} \sum_{\nu_{1}+\nu_{2}=\nu} x_{\nu_{1}}^{\left[k_{1}\right]} x_{\nu_{2}}^{\left[k_{2}\right]},
$$

while for $\nu=0$ we require

$$
\sum_{k_{1}+k_{2}=k} \sum_{\nu_{1}+\nu_{2}=\nu} x_{\nu_{1}}^{\left[k_{1}\right]} x_{\nu_{2}}^{\left[k_{2}\right]}=0
$$

By setting $c_{k}=x_{0}^{[k]}$ the latter equation can be written as [cf. (2.10)]

$$
c_{1}=0, \quad c_{k}=-\frac{1}{2 c_{0}} \sum_{k^{\prime}=1}^{k-1} \sum_{\nu \in Z} x_{\nu}^{\left[k-k^{\prime}\right]} x_{-\nu}^{\left[k^{\prime}\right]}, \quad k \geqslant 2 .
$$

Then we can proceed as in Sec. III, with some slight changes that we now explain. First of all note that (5.5) gives for $\nu \neq 0$,

$$
\begin{gathered}
x_{\nu}^{[0]}=0, \\
x_{\nu}^{[1]}=\frac{\varepsilon f_{\nu}}{i \omega \nu(1+i \varepsilon \omega \nu)},
\end{gathered}
$$




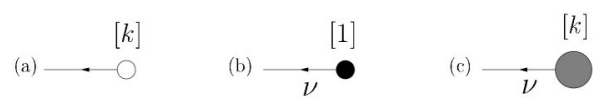

FIG. 10. Graphical representation of $x_{0}^{[k]}, x_{\nu}^{[1]}$, and $x_{\nu}^{[k]}$. For $\nu=0$ the latter reduces to the first graph, while for $k=1$ and $\nu \neq 0$ it reduces to the second graph. In the first graph the momentum is not shown as it is necessarily $\nu=0$.

$$
x_{\nu}^{[k]}=-\frac{\varepsilon}{i \omega \nu(1+i \varepsilon \omega \nu)} \sum_{k_{1}+k_{2}=k-1} \sum_{\nu_{1}+\nu_{2}=\nu} x_{\nu_{1}}^{\left[k_{1}\right]} x_{\nu_{2}}^{\left[k_{2}\right]}, \quad k \geqslant 2 .
$$

Then the graphical representations of $x_{0}^{[k]}, x_{\nu}^{[1]}$, and $x_{\nu}^{[k]}$ are as in the previous case, with the only change in the representation of the order labels (because of the square brackets instead of the parentheses); see Fig. 10.

On the contrary the graphical representation of the third equation in (5.8) is as in Fig. 11.

At the end we obtain a tree expansion where the trees differ from the previous ones as they contain no vertex with only one entering line. With the previous notations this means that $L_{1}(\theta)$ $=\varnothing$ and $V_{1}(\theta)=\varnothing$, hence $V(\theta)=V_{2}(\theta)$. Moreover also the propagators and the node factors of the vertices are different, as (3.1) and (3.2) must be replaced with

$$
g_{\ell}= \begin{cases}1 /\left(\left(i \omega \nu_{\ell}\right)\left(1+i \varepsilon \omega \nu_{\ell}\right)\right), & \nu_{\ell} \neq 0, \\ 1, & \nu_{\ell}=0,\end{cases}
$$

and, respectively,

$$
F_{v}= \begin{cases}-\varepsilon, & v \notin V_{0}(\theta), \\ -1 / 2 c_{0}, & v \in V_{0}(\theta),\end{cases}
$$

and we recall once more that only vertices $v$ with $s_{v}=2$ are allowed. Finally, the node factors associated with the endpoints are $F_{v}=c_{k_{v}}$ if $v$ is a white bullet and $F_{v}=\varepsilon f_{v_{v}}$ if $v$ is a black bullet.

As in Sec. III we can envisage an expansion in which all white bullets $v$ have $k_{v}=0$ (simply by expanding iteratively in trees the white bullets of higher order). A tree appearing in this new expansion is represented in Fig. 12.

With the notations (4.1), we obtain the bounds

$$
\begin{gathered}
\left.\prod_{v \in V(\theta) \backslash V_{0}(\theta)} F_{v}|\leqslant| \varepsilon\right|^{|V(\theta)|}, \quad\left|\prod_{\ell \in L(\theta)} g_{\ell}\right| \leqslant \prod_{\ell \in L_{2}(\theta)} \frac{1}{\left|\omega \nu_{\ell}\right|\left|1+i \varepsilon \omega \nu_{\ell}\right|}, \\
\left|\prod_{v \in V_{0}(\theta)} F_{v}\right| \leqslant\left(\frac{1}{2 c_{0}}\right)^{\left|V_{0}(\theta)\right|},\left|\prod_{v \in E_{W}(\theta)} F_{v}\right| \leqslant c_{0}^{\left|E_{W}(\theta)\right|},
\end{gathered}
$$

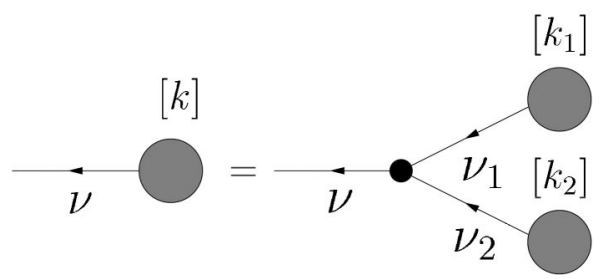

FIG. 11. Graphical representation of the second equation in (5.8) expressing the coefficient $x_{v}^{[k]}$ for $k \geqslant 2$ and $\nu \neq 0$ in terms of the coefficients $x_{\nu^{\prime}}^{\left[k^{\prime}\right]}$ with $k^{\prime}<k$. In the right-hand graph one has the constraints $k_{1}+k_{2}=k-1$ and $\nu_{1}+\nu_{2}=\nu$. 


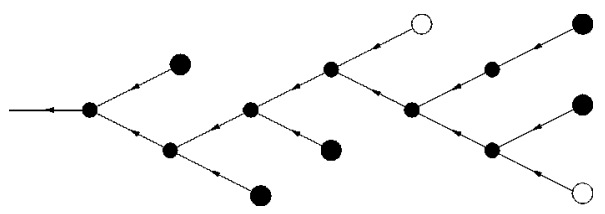

FIG. 12. Example of tree appearing in the new graphical expansion. The number of lines entering any vertex $v$ can be only $s_{v}=2$. The order of the tree is given by $|B(\theta)|-\left|V_{0}(\theta)\right|$. All the white bullets have order labels (0), and additionally all the black bullets carry a label (1); hence we can avoid drawing explicitly such labels.

$$
\left|\prod_{v \in E_{B}(\theta)} F_{v}\right| \leqslant F^{\left|E_{B}(\theta)\right|} \prod_{v \in E_{B}(\theta)} e^{-\xi\left|\nu_{v}\right|},
$$

where we have again used the bound $\left|f_{\nu}\right| \leqslant F e^{-\xi|\nu|}$, for suitable (strictly) positive constants $F$ and $\xi$, which follows from the analyticity assumption on $f$.

For real $\varepsilon$ we can bound each propagator by

$$
\left|g_{\ell}\right| \leqslant \frac{1}{\left|\omega \nu_{\ell}\right|} \leqslant \frac{1}{|\omega|}
$$

so that the value of any tree $\theta \in \mathcal{T}_{k, \nu}$ can be bounded by

$$
|\operatorname{Val}(\theta)| \leqslant|\varepsilon|^{k}|\omega|^{-k}\left(\max \left\{c_{0}, F\right\}\right)^{k}\left(\max \left\{1,1 / 2 c_{0}\right\}\right)^{k} \prod_{v \in E_{B}(\theta)} e^{-\xi\left|\nu_{v}\right|},
$$

where we have again used Lemma 4.1. If we write

$$
\prod_{v \in E_{B}(\theta)} e^{-\xi\left|\nu_{v}\right|} \leqslant e^{-\xi|\nu| / 2}\left(\prod_{v \in E_{B}(\theta)} e^{-\xi\left|\nu_{v}\right| / 2}\right)
$$

we can proceed as in Sec. IV; we use the last product to perform the sum over the Fourier labels, which gives a factor $B_{2}^{k}$, whereas the sum over the unlabeled trees gives a factor $2^{2 k-1}$. At the end we obtain

$$
\left|x_{\nu}^{(k)}\right| \leqslant \mu_{2}^{-k}, \quad\left|x^{(k)}(t)\right| \leqslant \mu_{2}^{-k},
$$

where we have set $\mu_{2}^{-1}=4|\omega|^{-1} \max \left\{1,1 / 2 c_{0}\right\} \max \left\{F B_{2}, c_{0}\right\}|\varepsilon|$. Hence the radius of convergence $\mu_{0}$ of the series expansion (5.4) is bounded as $\mu_{0} \geqslant \mu_{2}=O(1 /|\varepsilon|)$, so that for $\varepsilon$ small enough, say $|\varepsilon|<\varepsilon_{3}=\left(4|\omega|^{-1} \max \left\{1,1 / 2 c_{0}\right\} \max \left\{F B_{2}, c_{0}\right\}\right)^{-1}$, the value $\mu=1$ is inside the analyticity domain.

We can summarize the results found so far as follows.

Theorem 5.1: Given the equation (2.1) with $f$ analytic, there exists $\varepsilon_{0}>0$ such that for all real $\varepsilon$ with $|\varepsilon|<\varepsilon_{0}$ there is only one periodic solution which admits a formal expansion in powers of $\varepsilon$, and the corresponding period is the same period $2 \pi / \omega$ as the forcing term. An explicit bound is $\varepsilon_{0} \geqslant \varepsilon_{3}=O(\omega)$.

Note that if $\omega$ is very large then very large values of $\varepsilon$ are allowed.

We can investigate further the regularity properties in $\varepsilon$ of the periodic solution found in Theorem 5.1, and see what happens for complex values of $\varepsilon$.

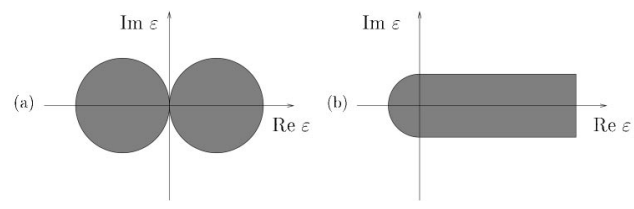

FIG. 13. (a) Region $\mathcal{C}_{R}$ in the complex $\varepsilon$-plane and (b) striplike region of analyticity $\mathcal{S}_{B}$ of the Borel transform. The region $\mathcal{C}_{R}$ is the union of two discs of radius $R / 2$ and centers $( \pm R / 2,0)$. 
We need the following preliminary result [see Fig. 13(a) for the region $\mathcal{C}_{R}$ ].

Lemma 5.2: Given $\omega>0$ and $0<R<1 / 4 \omega$ let $\mathcal{C}_{R}$ be the pair of discs $\mathcal{C}_{R}=\left\{\varepsilon:\left|\operatorname{Re} \varepsilon^{-1}\right|\right.$ $\left.>R^{-1}\right\}$. For all $\varepsilon \in \mathcal{C}_{R}$ and all $\nu \in \mathbb{Z} \backslash\{0\}$ one has $|i \omega \nu(1+i \varepsilon \omega \nu)| \geqslant \omega / 2$.

Proof: Write $\varepsilon=a+i b$ and $x=\omega \nu$, so that one has $|i \omega \nu(1+i \varepsilon \omega \nu)|=|x| \sqrt{(1-b x)^{2}+(a x)^{2}} \equiv F(x)$. If $\varepsilon \in \mathcal{C}_{R}$ one has $|a| \geqslant b^{2} / 2 R$. Fix $0<A<1$. If $|1-b x| \leqslant A$ then $\sqrt{(1-b x)^{2}+(a x)^{2}} \geqslant|a x|$ $\geqslant b^{2}|x| / 2 R \geqslant|b|(1-A) / 2 R$, so that $F(x) \geqslant(1-A)^{2} / 2 R$. If $|1-b x| \geqslant A$ then $\sqrt{(1-b x)^{2}+(a x)^{2}} \geqslant A$, hence $F(x) \geqslant A|x| \geqslant \omega A$. Then choose $A=1-\sqrt{\omega R} \geqslant 1 / 2$; this gives $F(x) \geqslant \omega / 2$.

Now fix $0<R<\bar{R} \equiv \varepsilon_{3}$ so small that $|\omega| R<1 / 4$, and consider the corresponding domain $\mathcal{C}_{R}$. We can apply Lemma 5.2 and deduce that any propagator $g_{\ell}$ is bounded by $\left|g_{\ell}\right| \leqslant 2 /|\omega|$ for all $\varepsilon \in \mathcal{C}_{R}$.

This allows us to obtain the following result.

Proposition 5.3: There exists $R>0$ small enough such that in the domain $\mathcal{C}_{R}$ one has the asymptotic expansion

$$
x(t)=\sum_{k=0}^{N-1} \varepsilon^{k} x^{(k)}(t)+\mathfrak{R}_{N}(\varepsilon), \quad\left|\Re_{N}(\varepsilon)\right| \leqslant A B^{N} N !|\varepsilon|^{N},
$$

where the constants $A$ and $B$ are uniform in $N$ and in $\varepsilon$.

Proof: Write $x(t)$ as $x(t)=x_{N}(t)+\mathfrak{R}_{N}(t)$, where $x_{N}(t)$ is given by the sum of the first $N-1$ orders of the formal power series expansion of the solution $x(t)$ as in (5.16). For $\varepsilon \in \mathcal{C}_{R}$ the function (5.4) with $\mu=1$ is $C^{\infty}$ in $\varepsilon$, hence we can estimate $\mathfrak{R}_{N}(\varepsilon)$ with a bound on the $N$ th derivative of $x(t)$ in $\mathcal{C}_{R}$, and this gives the bound in (5.16).

Of course the constants $A$ and $B$ in (5.16) are explicitly computable; in particular one finds $B=O\left(\varepsilon_{3}^{-1}\right)$.

Then we are under the assumptions where Nevanlinna's theorem ${ }^{11}$ (see also Ref. 12) can be applied, and hence the series for

$$
\mathfrak{B}(t ; \varepsilon)=\sum_{k=0}^{\infty} \frac{1}{k !} \varepsilon^{k} x^{(k)}(t)
$$

converges for $|\varepsilon|<B$ [with $B$ given in (5.16)] and has an analytic continuation to $\mathcal{S}_{B}$ $=\left\{\varepsilon: \operatorname{dist}\left(\varepsilon, \mathbb{R}_{+}\right)<B\right\}$ [see Fig. 13(b)], satisfying for some constant $K$ the bound $|\mathfrak{B}(t ; \varepsilon)|$ $\leqslant K e^{|\varepsilon| R}$ uniformly in every $\mathcal{S}_{B^{\prime}}$ with $B^{\prime}<B$. The function $x(t)$ can be represented as the absolutely convergent integral

$$
x(t)=\frac{1}{\varepsilon} \int_{0}^{\infty} e^{-s / \varepsilon} \mathfrak{B}(t ; s) \mathrm{d} s
$$

for all $\varepsilon \in \mathcal{C}_{R}$, and this property can be stated by saying that $x(t)$ is Borel summable (in $\varepsilon$ ) and $\mathfrak{B}(t ; \varepsilon)$ is its Borel transform. ${ }^{9}$ This implies that the function given by the summation procedure described in Theorem 1 is unique. Therefore we have obtained the following result, which strengthens Theorem 1.

Theorem 5.4: The solution given by Theorem 1 is Borel summable at the origin.

Note that Watson's theorem cannot be invoked to obtain this result because the singularities are along the imaginary axis.

In particular if $f(\omega t)=\alpha+\beta \sin t$ then there is a periodic solution $x(t)=a+\varepsilon \beta \cos t+O\left(\varepsilon^{2}\right)$, with $a=\sqrt{\alpha}$, which has period $2 \pi$ and moves around the fixed point $(x, \dot{x})=(a, 0)$, and close to it within $O(\varepsilon)$. No other periodic solution analytic in $\varepsilon$ can exist.

We conclude this section with two remarks. The summation criterion envisaged in this section is reminiscent of that used (in a more difficult situation) in Ref. 6 for hyperbolic lowerdimensional tori. However in that case we are not able to prove Borel summability because to order $k$ the bounds were like $(k !)^{\alpha}$ for some $\alpha>1$. Neither extension to Watson's theorem ${ }^{9}$ 
analogous to the Nevanlinna-Sokal result (as those developed in Ref. 5) can be used because the exponent $\alpha$ is too large. We shall find a very similar situation in next section.

The lack of analyticity in $\varepsilon$ in a neighborhood of the origin is due to the accumulation of singularity points along the imaginary axis in the complex $\varepsilon$-plane (where the quantity $1+i \varepsilon \omega \nu$ vanishes for $\nu \in \mathbb{Z}$ ). The analyticity domain is tangential to the imaginary axis, and this allows us to apply Nevanlinna's theorem. We find that this situation has some analogies with a different problem, the analyticity properties of rescaled versions of some dynamical systems, such as Siegel's problem ${ }^{4}$ (and its linearization as considered in Ref. 10), the standard map ${ }^{2}$ and generalized standard maps, ${ }^{3}$ for complex rotation numbers tending to rational values in the complex plane. In those cases, however, only nontangential limits could be considered. Of course the situation is slightly more complicated there, because the set of accumulating singularity points is dense- and not only numerable as in the present case.

\section{QUASIPERIODIC FORCING TERMS}

In the case of analytic quasiperiodic forcing terms, we shall assume a Diophantine condition on the rotation vector $\boldsymbol{\omega}$, that is

$$
|\boldsymbol{\omega} \cdot \boldsymbol{\nu}| \geqslant C_{0}|\boldsymbol{\nu}|^{-\tau} \quad \forall \boldsymbol{\nu} \in \mathbb{Z}^{d} \backslash\{\mathbf{0}\},
$$

where $|\boldsymbol{\nu}|=|\boldsymbol{\nu}|_{1} \equiv\left|\nu_{1}\right|+\cdots+\left|\nu_{d}\right|$, and $C_{0}$ and $\tau$ are positive constants. We need $\tau \geqslant d-1$ in order to have a nonvoid set of vectors satisfying the condition (6.1), and $\tau>d-1$ in order to have a full measure set of such vectors. For simplicity (and without loss of generality) we can assume $C_{0}$ $<\gamma / 2$, with $\gamma=\min \{1,|c|\}$, where $c$ is a suitable constant to be fixed as $c=-2 c_{0}$, with $c_{0}=\sqrt{\alpha}$.

The equation of motion can be written in Fourier space as

$$
i \boldsymbol{\omega} \cdot \boldsymbol{\nu}(1+i \varepsilon \boldsymbol{\omega} \cdot \boldsymbol{\nu}) x_{\boldsymbol{\nu}}+\varepsilon \sum_{\boldsymbol{\nu}_{1}+\boldsymbol{\nu}_{2}=\boldsymbol{\nu}} x_{\boldsymbol{\nu}_{1}} x_{\boldsymbol{\nu}_{2}}=\varepsilon f_{\boldsymbol{\nu}},
$$

and the formal expansion for a quasiperiodic solution with frequency vector $\boldsymbol{\omega}$ reads as

$$
x(t)=\sum_{k=0}^{\infty} \varepsilon^{k} x^{(k)}(t)=\sum_{k=0}^{\infty} \varepsilon^{k} \sum_{\boldsymbol{\nu} \in \mathbb{Z}^{d}} e^{i \boldsymbol{\nu} \cdot \boldsymbol{\omega} t} x_{\boldsymbol{\nu}}^{(k)}
$$

and to see that the coefficients $x_{\boldsymbol{v}}^{(k)}$ are well defined to all orders $k \geqslant 0$ one can proceed as in Sec. II, with no extra difficulty. In particular the Diophantine condition (6.1) is sufficient to assure analyticity in $t$ of the coefficients $x^{(k)}(t)$.

Also the graphical representation can be worked out as in Sec. III. The only difference is that now the propagators of the lines with nonvanishing momentum $\boldsymbol{\nu}_{\ell}$, which is defined according to (3.2), with the vectors replacing the scalars, are given by $1 /\left(i \boldsymbol{\omega} \cdot \boldsymbol{\nu}_{\ell}\right)$, the node factors associated with the vertices $v$ with $s_{v}=1$ are given by $F_{v}=-\left(i \boldsymbol{\omega} \cdot \boldsymbol{\nu}_{\ell_{v}}\right)^{2}$, and the node factors associated with the black bullets $v$ are given by $F_{v}=f_{\boldsymbol{\nu}_{v}}$, with $\boldsymbol{\nu}_{v} \in Z^{d} \backslash\{\mathbf{0}\}$. All the other notations remain unchanged.

This yields that the propagators and the node factors can be bounded as in (4.2) and (4.3), with just a few differences of notation. More precisely one has

$$
\begin{gathered}
\left|\prod_{v \in V_{1}(\theta)} F_{v}\right|\left|\prod_{\ell \in L_{1}(\theta)} g_{\ell}\right| \leqslant \prod_{\ell \in L_{1}(\theta)}|\boldsymbol{\omega}|\left|\boldsymbol{\nu}_{\ell}\right|, \quad\left|\prod_{\ell \in L_{1}(\theta)} g_{\ell}\right| \leqslant \prod_{\ell \in L_{1}(\theta)} \frac{1}{\left|\boldsymbol{\omega} \cdot \boldsymbol{\nu}_{\ell}\right|} \leqslant C_{0}^{-1}\left|\boldsymbol{\nu}_{\ell}\right|^{\tau}, \\
\left|\prod_{v \in V_{0}(\theta)} F_{v}\right| \leqslant\left(\frac{1}{2 c_{0}}\right)^{\left|V_{0}(\theta)\right|},\left|\prod_{v \in E_{W}(\theta)} F_{v}\right| \leqslant c_{0}^{\left|E_{W}(\theta)\right|},
\end{gathered}
$$




$$
\left|\prod_{v \in E_{B}(\theta)} F_{v}\right| \leqslant F^{\left|E_{B}(\theta)\right|} \prod_{v \in E_{B}(\theta)} e^{-\xi\left|\boldsymbol{\nu}_{v}\right|},
$$

where the only bound which introduces a real difficulty with respect to the case of periodic forcing terms is the second one in the first line. Indeed it is the source of a small divisors problem, which cannot be set only through the Diophantine condition (6.1).

To each order $k$ we obtain for $x^{(k)}(t)$ a bound like $A B^{k} k !^{\max \{1, \tau\}}$, where the factor 1 arises from the propagators of the lines in $L_{1}(\theta)$ and the factor $\tau$ from those of the lines in $L_{2}(\theta)$ in (6.3). The last assertion is easily proved by reasoning as in (4.3), with $\max \left\{|\boldsymbol{\omega}|\left|\boldsymbol{\nu}_{\ell}\right|, C_{0}^{-1}\left|\boldsymbol{\nu}_{\ell}\right| \tau\right\}$ $\leqslant \max \left\{C_{0}^{-1},|\boldsymbol{\omega}|\right\}\left|\boldsymbol{\nu}_{\ell}\right|^{\max \{1, \tau\}}$ replacing $\nu_{\ell}$. In particular only for $d=2$ and $\tau=1$ we obtain the same bound proportional to $k$ ! as in the case of periodic solution (of course with different constants $A$ and $B$ ). Note that the vectors satisfying the Diophantine condition (6.1) with $\tau=1$ for $d=2$ is of zero measure but everywhere dense. An example of vector of this kind is $\boldsymbol{\omega}=\left(1, \gamma_{0}\right)$, where $\gamma_{0}$ $=(\sqrt{5}-1) / 2$ is the golden section.

However, to deal with the problem of accumulation of small divisors and discuss the issue of convergence of the series, we need renormalization group techniques. The first step is just to introduce a multiscale decomposition of the propagators, and this leads naturally to the introduction of clusters and self-energy graphs into the trees. The discussion can be performed either as in Ref. 6 or as in Ref. 8 (and in Ref. 7). We choose to follow Ref. 8, which is more similar to the present problem because the propagators are scalar quantities and not matrices. In any case, with respect to the quoted reference, we shall use a multiscale decomposition involving only the quantities $\left|\boldsymbol{\omega} \cdot \boldsymbol{\nu}_{\ell}\right|$, that is without introducing any dependence on $\varepsilon$ in the compact support functions. Indeed this is more suitable to investigate the analyticity properties in $\varepsilon$, and, as we shall see, we shall not need to exclude any real value of $\varepsilon$ in order to give a meaning to the resummed series, a situation more reminiscent of Ref. 6 than of Ref. 8 .

In the following we confine ourselves to outlining the main differences with respect to Ref. 8 . Let us introduce the functions $\psi_{n}$ and $\chi_{n}$, for $n \geqslant 0$, as in Ref. 8, Sec. 5. In particular $\psi_{n}(|x|) \neq 0$ implies $|x| \geqslant 2^{-(n+1)} C_{0}$ and $\chi_{n}(|x|) \neq 0$ implies $|x| \leqslant 2^{-n} C_{0}$. We shall define recursively the renormalized propagators $g_{\ell}^{[n]}=g^{[n]}\left(\boldsymbol{\omega} \cdot \boldsymbol{\nu}_{\ell} ; \varepsilon\right)$ and the counterterms $\mathcal{M}^{[n]}(\boldsymbol{\omega} \cdot \boldsymbol{\nu} ; \varepsilon)$ on scales $n$ as

$$
\begin{gathered}
g^{[-1]}(x ; \varepsilon)=1, \quad M^{[-1]}(x ; \varepsilon)=0, \\
g^{[0]}(x ; \varepsilon)=\frac{\psi_{0}(|x|)}{i x(1+i \varepsilon x)}, \quad M^{[0]}(x ; \varepsilon)=\sum_{k=1}^{\infty} \sum_{T \in \mathcal{S}_{k, 0}^{\mathcal{R}}} \mathcal{V}_{T}(x ; \varepsilon), \\
g^{[n]}(x ; \varepsilon)=\frac{\chi_{0}(|x|) \cdots \chi_{n-1}(|x|) \psi_{n}(|x|)}{i x(1+i \varepsilon x)+\mathcal{M}^{[n-1]}(x ; \varepsilon)}, \\
\mathcal{M}^{[n]}(x ; \varepsilon)=\mathcal{M}^{[n-1]}(x ; \varepsilon)+\chi_{0}(|x|) \cdots \chi_{n-1}(|x|) \chi_{n}(|x|) M^{[n]}(x ; \varepsilon), \\
M^{[n]}(x ; \varepsilon)=\sum_{k=1}^{\infty} \sum_{T \in \mathcal{S}_{k, n}^{\mathcal{R}}} \mathcal{V}_{T}(x ; \varepsilon),
\end{gathered}
$$

where the set of renormalized self-energy graphs $\mathcal{S}_{k, n}^{\mathcal{R}}$ and the self-energy graphs $\mathcal{V}_{T}(x ; \varepsilon)$ are defined as in Ref. 8, Sec. 6. We have explicitly used the fact that the first contribution to the self-energy graphs is of order $k=1$ (see Fig. 14). Note that one has $\chi_{0}(|x|) \cdots \chi_{n-1}(|x|) \chi_{n}(|x|)$ $=\chi_{n}(|x|)$, so that if $g^{[n]}(x ; \varepsilon) \neq 0$ then one has $2^{-(n+1)} C_{0} \leqslant|x| \leqslant 2^{-(n-1)} C_{0}$.

Then one defines for $k \geqslant 1$ 


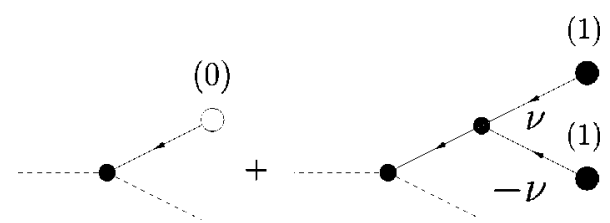

FIG. 14. Lower order contributions to the counterterm arising from self-energy graphs of order $k=1$ and $k=3$. The (dashed) external lines do not enter into the definition of self-energy graph, and they have been drawn only with the aim of helping to visualize the structure of the self-energy graph.

$$
x_{\boldsymbol{\nu}}^{[k]}=\sum_{\theta \in \mathcal{T}_{k, \boldsymbol{\nu}}} \operatorname{Val}(\theta), \quad x_{\mathbf{0}}^{[k]} \equiv c_{k}=\sum_{\theta \in \mathcal{T}_{k, \mathbf{0}}}{ }^{*} \operatorname{Val}(\theta),
$$

where the tree value is defined as

$$
\operatorname{Val}(\theta)=\left(\prod_{\ell \in L(\theta)} g_{\ell}^{\left[n_{\ell}\right]}\right)\left(\prod_{v \in E(\theta) \cup V(\theta)} F_{v}\right),
$$

and, as before, * means that there are two lines entering the last vertex $v_{0}$ of $\theta_{0}$ and neither one exits from an endpoint $v$ with order label $k_{v}=0$. For $k=1$ the second of (6.7) must be interpreted as $c_{1}=0$.

Furthermore one has

$$
\begin{gathered}
M^{[0]}(x ; \varepsilon)=M^{[0]}(0 ; \varepsilon)+O\left(\varepsilon^{2} x\right), \\
M^{[0]}(0 ; \varepsilon)=-2 \varepsilon c_{0}+M_{2}^{[0]}(0 ; \varepsilon), \quad M_{2}^{[0]}(0 ; \varepsilon)=O\left(\varepsilon^{2}\right),
\end{gathered}
$$

and an easy computation shows (cf. Fig. 14) that

$$
M_{2}^{[0]}(0 ; \varepsilon)=\varepsilon^{3} \frac{1}{c_{0}} \sum_{\boldsymbol{\nu} \neq \mathbf{0}} \psi_{0}^{2}(|\boldsymbol{\omega} \cdot \boldsymbol{\nu}|) \frac{\left|f_{\boldsymbol{\nu}}\right|^{2}}{(\boldsymbol{\omega} \cdot \boldsymbol{\nu})^{2}\left(1+(\varepsilon \boldsymbol{\omega} \cdot \boldsymbol{\nu})^{2}\right)}+O\left(\varepsilon^{4}\right),
$$

so that in fact one has $M_{2}^{[0]}(0 ; \varepsilon)=O\left(\varepsilon^{3}\right)$.

Moreover to higher scales one has $M^{[n]}(x ; \varepsilon)=M^{[n]}(0 ; \varepsilon)+O\left(\varepsilon^{3} x\right)$, with

$$
M^{[n]}(0 ; \varepsilon)=-\varepsilon^{3} \frac{1}{c_{0}} \sum_{\boldsymbol{\nu} \neq \mathbf{0}} \sum_{n_{1}+n_{2}=n} \psi_{n_{1}}(|\boldsymbol{\omega} \cdot \boldsymbol{\nu}|) \psi_{n_{2}}(|\boldsymbol{\omega} \cdot \boldsymbol{\nu}|) \frac{\left|f_{\nu}\right|^{2}}{(\boldsymbol{\omega} \cdot \boldsymbol{\nu})^{2}\left(1+(\boldsymbol{\omega} \cdot \boldsymbol{\nu})^{2}\right)}+O\left(\varepsilon^{4}\right)
$$

so that each $M^{[n]}(0 ; \varepsilon)$ is a higher order correction to $M^{[0]}(0 ; \varepsilon)$ and it decays exponentially in $n$ (because of the compact support functions).

The following result holds.

Lemma 6.1: Assume that the renormalized propagators up to scale $n-1$ can be bounded as

$$
\left|g_{\ell}^{\left[n_{\ell}\right]}\right| \leqslant C_{1}^{-1} 2^{\beta n_{\ell}}
$$

for some positive constants $C_{1}$ and $\beta$. Then for all $n^{\prime} \leqslant n-1$ the number $N_{n^{\prime}}(\theta)$ of lines on scale $n^{\prime}$ in $\theta$ is bounded by

$$
N_{n^{\prime}}(\theta) \leqslant K 2^{-n^{\prime} / \tau} \sum_{v \in E_{B}(\theta)}\left|\boldsymbol{\nu}_{v}\right|
$$

for some positive constant $K$. If $|\varepsilon|<\varepsilon_{0}$, with $\varepsilon_{0}$ small enough, then for all $n^{\prime} \leqslant n$ one has

$$
\left|M^{\left[n^{\prime}\right]}(x ; \varepsilon)\right| \leqslant D_{1}|\varepsilon|^{3} e^{-D_{2} 2^{n^{\prime} / \tau}}, \quad\left|\partial_{x} M^{\left[n^{\prime}\right]}(x ; \varepsilon)\right| \leqslant D_{1}|\varepsilon|^{3} e^{-D_{2} 2^{n^{\prime} / \tau}},
$$



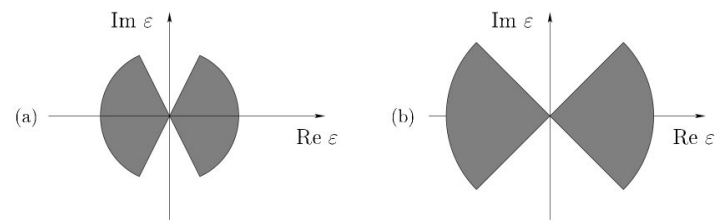

FIG. 15. Region $\mathcal{D}_{R, \lambda}$ in the complex $\varepsilon$-plane for $\lambda=\tan \pi / 6$ (a) and for $\lambda=1$ (b). One can write $\lambda=\tan \varphi$, where $\varphi$ is the angle between the imaginary axis and the line $a=\lambda b$.

for some $C_{1}$-independent positive constants $D_{1}$ and $D_{2}$. Only the constant $D_{1}$ depends on $\beta$. The constant $\varepsilon_{0}$ can be written as $\varepsilon_{0}=C_{1} C_{2}^{-\beta} C_{3}$, with $C_{2}$ and $C_{3}$ two positive constants independent of $\beta$ and $C_{1}$.

Proof: The proof can be easily adapted from the proofs of Lemma 1 and Lemma 2 of Ref. 8 .

So we are left with the problem of proving that the renormalized propagators satisfy the bounds (6.11). To this end let us introduce the notation

$$
F(x)=F_{0}(x)+c_{1}(\varepsilon) \varepsilon+c_{2}(\varepsilon, x) \varepsilon^{2} x, \quad F_{0}(x)=i x(1+i \varepsilon x),
$$

with $x=\boldsymbol{\omega} \cdot \boldsymbol{\nu}$ and the functions $c_{1}(\varepsilon)$ and $c_{2}(\varepsilon, x)$ such that $c_{1}(\varepsilon)=c+c_{3}(\varepsilon) \varepsilon$, with $c \neq 0$, and the functions $\left|c_{2}(\varepsilon, x)\right|$ and $\left|c_{3}(\varepsilon)\right|$ bounded by a constant $c^{\prime}$ uniformly (in $\varepsilon$ and $x$ ). Recall that $\gamma$ $=\min \{1,|c|\}$ and $C_{0}<\gamma / 2$.

Fix $\lambda \in[0,1]$. Set $\mathcal{B}_{R}(0)=\{\varepsilon \in \mathrm{C}:|\varepsilon|<R\}$ and $\mathcal{D}_{R, \lambda}=\left\{\varepsilon=a+i b \in \mathcal{B}_{\lambda R}(0):|a| \geqslant \lambda|b|\right\}$ (see Fig. 15). The following result refines Lemma 5.2.

Lemma 6.2: Given $0<R<1 / 4 C_{0}$, let $\mathcal{C}_{R}$ be defined as in Lemma 5.2. For all $\varepsilon \in \mathcal{C}_{R}$ and all $x$ one has $\left|F_{0}(x)\right| \geqslant \min \left\{C_{0},|x|\right\} / 2$, while for all $\varepsilon \in \mathcal{D}_{R, \lambda}$ one has $\left|F_{0}(x)\right| \geqslant \lambda|x| / 2$.

Proof: Write $\varepsilon=a+i b$, so that $\left|F_{0}(x)\right|=|x| \sqrt{(1-b x)^{2}+(a x)^{2}}$. For $\varepsilon \in \mathcal{C}_{R}$ set $A=1-\sqrt{C_{0} R}$. If $|x|$ $\geqslant C_{0}$, for $|1-b x| \leqslant A$ one has $\left|F_{0}(x)\right| \geqslant\left|a x^{2}\right| \geqslant b^{2} x^{2} / 2 R \geqslant C_{0} / 2$, while for $|1-b x| \geqslant A$ one has $\left|F_{0}(x)\right| \geqslant A|x| \geqslant|x| / 2 \geqslant C_{0} / 2$. If $|x| \leqslant C_{0}$, for $|1-b x| \leqslant A$ one has $\left|F_{0}(x)\right| \geqslant\left|a x^{2}\right| \geqslant C_{0} / 2 \geqslant|x| / 2$, while for $|1-b x| \geqslant A$ one has $\left|F_{0}(x)\right| \geqslant A|x| \geqslant|x| / 2$. For $\varepsilon \in \mathcal{D}_{R, \lambda}$ set $A=1 / 2$, one finds $\left|F_{0}(x)\right|$ $\geqslant \lambda|x| / 2$.

Then the following result holds.

Lemma 6.3: Set $x=\omega \cdot \boldsymbol{\nu}$ and assume $|x| \leqslant C_{0}$. Then if $R$ is small enough one has $|F(x)|$ $\geqslant \lambda \gamma|x| / 8$ for all $\varepsilon \in \mathcal{D}_{R, \lambda}$.

Proof: Set $F_{1}(x)=F_{0}(x)+c \varepsilon$ and $\varepsilon=a+i b$. Then $F_{1}(x)=i\left(x+b\left(c-x^{2}\right)\right)+a\left(c-x^{2}\right)$, and $|F(x)|$ $\geqslant\left|F_{1}(x)\right|-c^{\prime}|\varepsilon|^{2}(1+|x|)$. If $\left|x+b\left(c-x^{2}\right)\right| \geqslant|x| / 2$ and $|b c| \geqslant 4|x|$ one has $\left|F_{1}(x)\right| \geqslant|c| \sqrt{b^{2}+a^{2}} / 2$ $\equiv|c \varepsilon| / 2$, so that $|F(x)| \geqslant|c \varepsilon| / 4 \geqslant|c b| / 4 \geqslant|x|$. If $\left|x+b\left(c-x^{2}\right)\right| \geqslant|x| / 2$ and $|b c| \leqslant 4|x|$ one has $\left|F_{1}(x)\right| \geqslant \gamma \max \left\{\sqrt{x^{2}+a^{2}},|\varepsilon| / 4\right\} / 2$, so that $|F(x)| \geqslant \gamma \sqrt{x^{2}+a^{2}} / 4 \geqslant \gamma|x| / 4$. If $\left|x+b\left(c-x^{2}\right)\right| \leqslant|x| / 2$ one has $\left|b\left(c-x^{2}\right)\right| \geqslant|x| / 2$ and $|b c| \leqslant 3|x|$, which give $|\varepsilon|^{2} \leqslant 3|\varepsilon| \sqrt{a^{2}+x^{2}} / \gamma \leqslant 3 \lambda R(|a|+|x|) / \gamma$, and $\left|F_{1}(x)\right| \geqslant\left|a\left(c-x^{2}\right)\right| \geqslant\left|a\left(c-x^{2}\right)\right| / 2+(\lambda|x| / 2) / 2 \geqslant \gamma \lambda(|a|+|x|) / 4$, so that $|F(x)| \geqslant \gamma \lambda|x| / 8$.

Then we can come back to the bounds of the renormalized propagators, and prove the following result.

Lemma 6.4: If $R$ is small enough for all $n \geqslant 0$ and all $\varepsilon \in \mathcal{D}_{R, \lambda}$ the renormalized propagators $g^{[n]}(x ; \varepsilon)$ satisfy the bounds (6.11) with $\beta=1$ and $C_{1}=\lambda C_{4}$, with a $\lambda$-independent constant $C_{4}$.

Proof: The proof can be done by induction on $n$. For $n=0$ the bound is trivially satisfied by Lemma 6.2. Assuming that the bounds hold for all $n^{\prime}<n$ then we can apply Lemma 6.1 and deduce the bounds (6.13). In turn this implies that the renormalized propagators on scale $n$ can be written as $g^{[n]}(x ; \varepsilon)=1 / F(x)$, with $F(x)$ written as in (6.14) for $c=-2 c_{0}$ [cf. (6.8)], and for suitable functions $c_{1}(\varepsilon)$ and $c_{2}(\varepsilon, x)$, depending on $n$ and satisfying the properties listed after (6.14) for some $n$-independent constant $c^{\prime}$. Then by Lemma 6.3 the renormalized propagators $g^{[n]}(x ; \varepsilon)$ satisfy the same bounds $(6.11)$ with $C_{1}=O(\lambda)$ for $\varepsilon \in \mathcal{D}_{R, \lambda}$.

Of course for real $\varepsilon$ the bound (6.11) is trivially satisfied, with $C_{1}=2^{-1} C_{0}$. This follows from Lemma 6.4 with $\lambda=1$, but it is obvious independently of that result because one has $c_{1}(\varepsilon)=c \varepsilon$ $+O\left(\varepsilon^{2}\right)$, with $c=-2 c_{0} \in \mathbb{R}$. If we want to take also complex values of $\varepsilon$, we have analyticity in a 


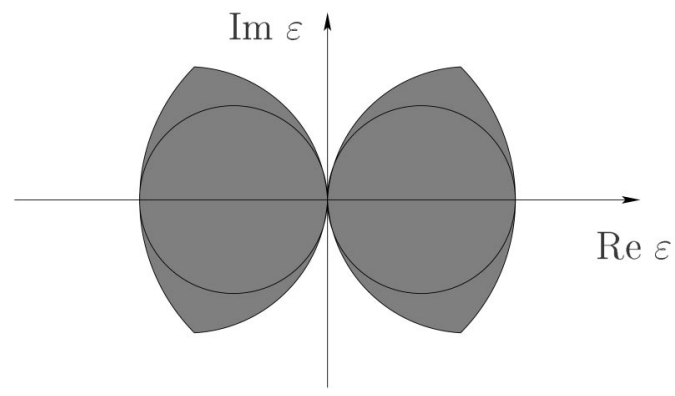

FIG. 16. Regions $\mathcal{D}_{R}$ and $\mathcal{C}_{R}$ in the complex $\varepsilon$-plane, $\mathcal{D}_{R}$ is the entire grey region, while $\mathcal{C}_{R}$ is the region contained inside the two circles.

domain $D$ which can be written as $\mathcal{D}_{R}=\cup_{\lambda \in[0,1]} \mathcal{D}_{R, \lambda}$. One can easily realize that the region $\mathcal{C}_{R}$ is contained inside the domain $\mathcal{D}_{R}$ (cf. Fig. 16). Fix $\lambda=\tan \varphi$, with $\varphi \in[0, \pi / 4]$ (see Fig. 15), for all such $\varphi$ the line which forms an angle $\varphi$ with the imaginary axis (see Fig. 15) and passes through the origin intersects the boundary of $\mathcal{D}_{R}$ at a distance $R \tan \varphi$ from the origin and the boundary of $\mathcal{C}_{R}$ at a distance $R \sin \varphi$. Hence we have an analyticity domain of the same form as in the case of periodic forcing terms. Nevertheless the results found so far do not allow us to obtain Borel summability, notwithstanding a circular analyticity domain $\mathcal{C}_{R}$ is found, as the bounds which are satisfied inside the region $\mathcal{C}_{R}$ are not uniform in $\varepsilon$ (because of the dependence on $\lambda$ ).

Note that $\beta=1$ in (6.11) is the same exponent appearing in the bounds of the propagators in the formal expansion. To obtain uniform bounds in a domain $\mathcal{C}_{R}$, for some value of $R$, we must allow larger values of $\beta$. The following result is obtained.

Lemma 6.5: Set $x=\omega \cdot \boldsymbol{\nu}$ and assume $|x|<C_{0}$. If $R$ is small enough one has $|F(x)|>\gamma|x|^{2} / 2$ for all $\varepsilon \in \mathcal{C}_{R}$.

Proof: Set $F_{1}(x)=F_{0}(x)+c \varepsilon$ and $\varepsilon=a+i b$. If $\left|x+b\left(c-x^{2}\right)\right| \leqslant|x| / 2$ one has $\left|b\left(c-x^{2}\right)\right| \geqslant|x| / 2$ and $3|x| \geqslant|b c| \geqslant|x| / 4$. Hence $\left|a\left(c-x^{2}\right)\right| \geqslant b^{2}\left|c-x^{2}\right| / 2 R \geqslant|x|^{2} / 16 R|c|$, so that one has $\left|F_{1}(x)\right| \geqslant \mid a(c$ $\left.-x^{2}\right)|\geqslant| a c|/ 4+| a\left(c-x^{2}\right) \mid / 2 \geqslant \gamma\left(|a|+x^{2} / 16 R c\right) / 2$. On the other hand, one has $|\varepsilon|^{2}=a^{2}+b^{2} \leqslant a^{2}$ $+9 x^{2} / c^{2}$, so that $|F(x)| \geqslant\left|F_{1}(x)\right|-2 c^{\prime}|\varepsilon|^{2} \geqslant\left|F_{1}(x)\right| / 2 \geqslant \gamma x^{2} / 2$. The case $\left|x+b\left(c-x^{2}\right)\right| \geqslant|x| / 2$ can be discussed as in Lemma 6.3, and it gives $|F(x)| \geqslant \gamma|x| / 4$.

Then we can prove the following result by proceeding exactly as in the proof of Lemma 8 .

Lemma 6.6: If $R$ is small enough for all $n \geqslant 0$ and all $\varepsilon \in \mathcal{C}_{R}$ the renormalized propagators $g^{[n]}(x ; \varepsilon)$ satisfy the bounds (6.11) with $\beta=2$ and $C_{1}$ a suitable constant.

The advantage of Lemma 6.4 with respect to Lemma 6.6 is that the bound of $R$ is better, which means that the domain $\mathcal{C}_{R}$ contained inside $\mathcal{D}_{R}$ in the first case is larger than the domain $\mathcal{C}_{R}$ of the second case. The advantage of Lemma 6.6 is that it allows uniform bounds inside the corresponding domain $\mathcal{C}_{R}$ to be obtained. Nevertheless, because of the factor $\beta=2$, a bound $A B^{k} k !^{2 \tau}$ is obtained for the coefficients $x^{(k)}(t)$ of the formal solution, and a result analogous to Proposition 2 can be proved also for the present case, with $N !^{2 \tau}$ replacing $N !$; we do not give the details as the proof is identical. Hence the bounds that we have are not good enough to obtain Borel summability in the case of quasiperiodic forcing terms, a situation strongly reminiscent of that encountered in Ref. 6. In fact at best one can set $\tau=1$ for $d=2$ (which, as noted above, corresponds to a set of Diophantine vectors of zero measure but everywhere dense), but this in turn implies a bound proportional to $N !^{2}$, which is not enough to apply Nevanlinna's theorem.

The conclusion is that the resummed series

$$
x(t)=\sum_{k=0}^{\infty} \mu^{k} x^{[k]}(t),
$$

where the coefficients $x^{[k]}(t)$ are given by 


$$
x^{[k]}(t) \sum_{\boldsymbol{\nu} \in \mathbb{Z}^{d}} e^{i \boldsymbol{\nu} \cdot \boldsymbol{\omega} t} x_{\mathbf{v}}^{[k]},
$$

with $x_{\nu}^{[k]}$ defined by (6.6), is well defined and converges. In general it is not obvious-even if expected-that (6.15) solves the equation of motion (1.1). Indeed, unlike the case of periodic forcing terms, we have no result, such as Nevanlinna's theorem on Borel summability, which we can rely upon in order to link the resummed series to the formal series. Therefore we must check by hand that by expanding in powers of $\varepsilon$ the resummed series we recover the formal power series (6.3). This means that the resummed series, which in principle could be unrelated to the equation of motion (because of the way it has been defined), in fact solves such an equation. Such a property can be proved by reasoning as in Ref. 8, Sec. 8. Again we omit the details, which can be easily worked out.

We can summarize our results in the following statement.

Theorem 6.7: Given the equation (1.1) with $f$ analytic in its argument and $\boldsymbol{\omega}$ satisfying the Diophantine condition (6.1), there exists $\varepsilon_{0}$ such that for all real $\varepsilon$ with $|\varepsilon|<\varepsilon_{0}$ there is a quasiperiodic solution with the same frequency vector as the forcing term. Such a solution extends to a function analytic in the domain $\mathcal{D}_{R}$ shown in Fig. 16, with $R=\varepsilon_{0}$.

The conclusion is that the summation criterion described here gives a well defined function, which is quasiperiodic and solves the equation of motion (1.1), but the criterion is not equivalent to Borel summability any more. In particular the issue of whether such quasiperiodic solutions are unique or not remains open, as in Ref. 6.

\section{EXTENSION TO MORE GENERAL NONLINEARITIES}

When considering the equation (1.5) the formal analysis of Sec. II (and of Sec. VI in the case of quasiperiodic forcing terms) can be performed essentially in the same way. If we write

$$
\begin{gathered}
g(x)=\sum_{p=0}^{\infty} \frac{1}{p !} g_{p}\left(x-c_{0}\right)^{p}, \quad g_{p}=\frac{\mathrm{d}^{p} g}{\mathrm{~d} x^{p}}\left(c_{0}\right), \\
{[g(x)]_{\nu}^{(k)}=\sum_{p=0}^{\infty} \frac{1}{p !} g_{p} \sum_{\substack{k_{1}+\cdots+k_{p}=k \\
\nu_{1}+\cdots+\nu_{p}=\nu}} x_{\boldsymbol{\nu}_{1}}^{\left(k_{1}\right)} \cdots x_{\boldsymbol{v}_{p}}^{\left(k_{p}\right)}, \quad k \geqslant 0,}
\end{gathered}
$$

then the recursive equations for $\boldsymbol{\nu} \neq \mathbf{0}$ are

$$
\begin{gathered}
x_{\boldsymbol{\nu}}^{(0)}=0, \\
x_{\boldsymbol{\nu}}^{(1)}=\frac{f_{\boldsymbol{\nu}}}{i \boldsymbol{\omega} \cdot \boldsymbol{\nu}}, \\
x_{\boldsymbol{\nu}}^{(k)}=-(i \boldsymbol{\omega} \cdot \boldsymbol{\nu}) x_{\boldsymbol{\nu}}^{(k-1)}-\frac{1}{i \boldsymbol{\omega} \cdot \boldsymbol{\nu}}[g(x)]_{\boldsymbol{\nu}}^{(k-1)}, \quad k \geqslant 2,
\end{gathered}
$$

while the compatibility condition becomes $[g(x)]_{0}^{(k)}=f_{\mathbf{0}} \delta_{k, 0}$ for $k \geqslant 0$. The latter for $k=0$ gives $g\left(c_{0}\right)=f_{0}$, while for $k \geqslant 1$ gives $g^{\prime}\left(c_{0}\right) c_{k}+R\left(c_{0}, c_{1}, \ldots, c_{k-1}\right)=0$, where the function $R\left(c_{0}, c_{1}, \ldots, c_{k-1}\right)$ depends on the coefficients to all orders $k^{\prime}<k$, hence, in particular, on the constants $c_{0}, \ldots, c_{k-1}$. Therefore the constants $c_{k}$ can be fixed iteratively as 


$$
c_{k}=-\frac{1}{g^{\prime}\left(c_{0}\right)} R\left(c_{0}, c_{1}, \ldots, c_{k-1}\right),
$$

provided that one has $g^{\prime}\left(c_{0}\right) \neq 0$, so that under the conditions (1.6) one has the formal solubility of the equations of motion (1.1). Note that the first condition in (1.6) requires $f_{\mathbf{0}} \in \operatorname{Ran}(g)$, and if such a condition is satisfied then the condition on the derivative is a genericity condition. Note also that the class of functions $g(x)$ which are not allowed depends on $f$ (more precisely on its average $f_{0}$ ). For instance an explicit example of a function which does not satisfy (1.6) is $g(x)=3 x^{2}-2 x^{3}$ if $f_{0}=1$.

The graphical representation differs from that of the preceding sections as now the number of lines entering a vertex $v$ can assume any value $s_{v} \in \mathbb{N}$, and if $v \notin V_{0}(\theta)$ the corresponding node factor is

$$
F_{v}=-\frac{\varepsilon}{s_{v} !} g_{s_{v}}
$$

which is bounded proportionally to some constant $G$ to the power $s_{v}$. Since $\Sigma_{v \in V(\theta)}\left(s_{v}-1\right)$ $=|E(\theta)|-1 \leqslant k-1$ (by Lemma 4.1) this produces an overall constant $G^{2 k}$ in the tree value. Also the study of the convergence of both the formal series and the resummed series can then be performed as in the previous case, and no further difficulty arises. The constant $c$ appearing after (6.14) becomes $-g^{\prime}\left(c_{0}\right)$, instead of $-2 c_{0}$, so that still one has $c \neq 0$ by the assumption (1.6).

\section{ACKNOWLEDGMENT}

The authors are indebted to Ugo Bessi for many enlightening discussions.

${ }^{1}$ Bartuccelli, M. V., Deane, J. H. B., Gentile, G., and Marsh, L., "Invariant sets for the varactor equation" (unpublished).

${ }^{2}$ Berretti, A. and Gentile, G., "Scaling properties for the radius of convergence of a Lindstedt series: the standard map," J. Math. Pures Appl. 78, 159-176 (1999)

${ }^{3}$ Berretti, A. and Gentile, G., "Scaling properties for the radius of convergence of a Lindstedt series: generalized standard maps," J. Math. Pures Appl. 79, 691-713 (2000).

${ }^{4}$ Berretti, A., Marmi, S., and Sauzin, D., "Limit at resonances of linearizations of some complex analytic dynamical systems," Ergod. Theory Dyn. Syst. 20, 963-990 (2000).

${ }^{5}$ Caliceti, E., Grecchi, V., and Maioli, M., "The distributional Borel summability and the large coupling $\Phi^{4}$ lattice fields," Commun. Math. Phys. 104, 163-174 (1986); 113, 173(E)-176(E) (1987).

${ }^{6}$ Gallavotti, G. and Gentile, G., "Hyperbolic low-dimensional invariant tori and summation of divergent series," Commun. Math. Phys. 227, 421-460 (2002).

${ }^{7}$ Gentile, G. and Gallavotti, G., "Degenerate elliptic tori," Commun. Math. Phys. (to be published).

${ }^{8}$ Gentile, G., "Quasi-periodic solutions for two-level systems," Commun. Math. Phys. 242, 221-250 (2003).

${ }^{9}$ Hardy, G. H., Divergent Series (Clarendon, Oxford, 1949).

${ }^{10}$ Marmi, S. and Sauzin, D., "Quasianalytic monogenic solutions of a cohomological equation," Mem. Am. Math. Soc. $164 \mathrm{vi}+83$ (2003).

${ }^{11}$ Nevanlinna, F., "Zur Theorie der Asymptotischen Potenzreihen,” Ann. Acad. Sci. Fenn., Ser. A 12, 1-18 (1916).

${ }^{12}$ Sokal, A. D., “An improvement of Watson's theorem on Borel summability,” J. Math. Phys. 21, 261-263 (1980). 